\title{
Development of a Practical Power Transmission Line Inspection Robot Based on a Novel Line Walking Mechanism
}

\author{
Ludan Wang, Fei Liu, Zhen Wang, Shaoqiang Xu, Sheng Cheng, and Jianwei Zhang, Member, IEEE
}

\begin{abstract}
A mobile robot based on novel line-walking mechanism is proposed for inspecting power transmission lines. The novel mechanism enables the centroid of the robot to concentrate on the hip joint to minimize the drive torque of the hip joint and keep the robot stable when only one leg is hung on line. After reviewing of the line-walking mechanism, power line inspection robot is described in detail. The pose adjustment analysis is carried out to make sure that the robot will keep in stable state when rolling on the power transmission line which forms a catenary curve. The obstacle-navigation cycle of the designed inspection robot is composed of a single-support phase and a double-support phase. The centroid of the robot will be adjusted to shift to the other leg to start a new single-support phase. The feasibility of this concept is then confirmed by performing experiments with a simulated line environment.
\end{abstract}

\section{INTRODUCTION}

$\mathrm{T}$ HE purpose of power transmission line inspection tasks is to check the running state and find damages of high voltage power transmission lines equipments. Up to now, extra-high (EHV) voltage power transmission line equipment is still inspected manually by workers with a telescope on the ground. Sometimes, they have to climb the towers or ride in gondolas suspended on the overhead ground wires. These working modes have many disadvantages, such as long inspection cycle, high working intensity, huge expense and high danger. Performing the inspection by helicopters, as the other method, is high efficient compare to manually operation. Despite a higher efficiency the disadvantage of this method is that it is more expensive and climate-dependant. Since the early 1990s, many researchers have investigated in the development of inspection robots to assist people or to replace people on power transmission line site [1].

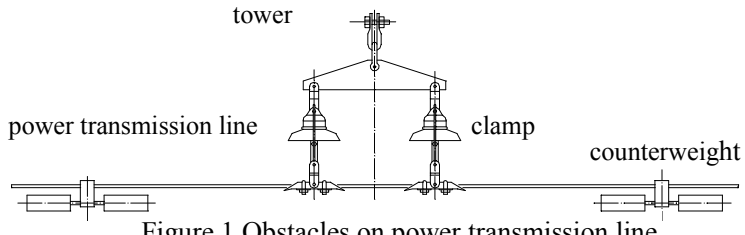

Figure 1 is conceptual sketch of obstacles close to the

Manuscript received March 10, 2010. This work was supported in part by the Shenzhen non-consensus fund project "Development of a line-walking robot".

Ludan Wang, Fei Liu, Zhen Wang, Shaoqiang Xu and Sheng Cheng work with the Laboratory of Intelligent Robot Engineering, KunShan Institute of Industrial Research. KunShan PRC. e-mail: 1dwang8078@sina.com; liufei@ksitri.com;; wangzhen@ksitri.com; xushaoqiang@ksitri.com; chengsheng@ksitri.com.

Jianwei Zhang works at *TAMS, University of Hamburg, Germany

e-mail: zhangjianwei@ksitri.com tower of power transmission line. One common approach to line-walking robot for fulfill power transmission line inspection task uses a conventional rail wheeled vehicle with the addition of a device that can overcome obstacles on the line. A wheeled trolley that carries an arc-shaped rail is described in [1], which by extending the rail to either side of the tower can be carried to the other side of obstacles. LineScout $[2,3,4]$, a line-walking robot developed at Hydro-Qu'ebec's research institute (IREQ), is a platform can cross obstacles by deploying a two-gripper auxiliary frame under the cable and securing a grasp on both sides of the obstacle. The traction wheels can then be released from the conductor, flipped down, and moved to the other side of the obstacle.

Generally, robots with structure mentioned above are relatively heavy since the wheeled system and obstacle-navigation device are developed separately. Articulated leg-wheel system, with compact configuration, help to provide enhanced locomotion capabilities on uneven terrain, thus legged structures with two or more wheels are predominant in the development of line-walking robot now days. The principal reason for articulations to enable the robot to accommodate and overcome most obstacles by permitting the correspond wheel to rise up when it collides with the obstacle. Tribrachiation mobile robots were presented in $[5,6$, 7], the multi-degree-of-freedom structure allows a minimum of two traction wheels to be secured to the cable. Technically, more than two legs provide redundant support and often increase the load capacity and safety. However, these benefits are achieved at the cost of increased complexity, size, and weight. As a result, the biped is the most common line-walking robot since it requires minimum joints and actuators to provide locomotion. The challenge is to provide adaptability to such robot so that they may surmount a variety of obstacles using limited resources.

During the obstacle-navigation process, like biped robot for ground motion, walking of line-walking robot is a periodic phenomena, the locomotion of biped robot can be divided into single-supported phase and double-supported phase respectively. In order to assist and obtain a stable motion in the single-supported phase it is important to either anchor the supporting arm to the line with gripper to resist the gravity or control the total center of gravity position beneath the supporting arm. A dual-arm robot designed for live-line inspection of extra-high-voltage is presented in [8], this platform is designed to hang from the OGW on its two wheeled arms, the control box containing electrical and electronic components can be shifted forward or backward to 
center the mass on either of the two arms, by which the robot is hanging to keep the horizon pose of the body and protect the line against the gripping forces. Due to additional counter-weight system, obstacle-navigation process of this robot is complex and time-consuming. Another two-arm vehicle developed in [9], by transferring a fair amount of its weight beneath the supporting arm so that the other arm can be raised and then pivoted to the other side of the obstacles. In contrast, this robot has potential to overcome obstacles within comparatively fewer steps since the rising locomotion of arm can be realized by adjusting center gravity position properly alone. But inherently requires a large space for the locomotion of counter-weight, which results in the less compact configuration. In [10], a novel tele-operated robot with an actuated arm that position a counter-weight is introduced, which by moving the counter-weight certain supporting point can be lifted to cross obstacles.

In [11] a novel mechanism for power transmission line inspection purpose was provided. Each leg of the robot has a prismatic joint to adjust the length of the leg and the centroid of the leg can be adjusted to the axis of the hip to reduce the energy consumption related to gravity. In this essay a practical power line inspection robot based on the line-walking mechanism was described. The structure of this paper is as follows. After a short review of the line-walking mechanism, section 2 describes the mechanical structure of the power line inspection robot; section 3 describes the pose adjustment process when the robot rolling on line, obstacle-navigation locomotion analysis is discussed in detail, in section 4 robot control and the experiment are presented for subsequent evaluation of the appropriate structure; finally section 5 presents the conclusion.

\section{MECHANICAL SYSTEM}

\section{A. Review of Line- Walking Mechanism}

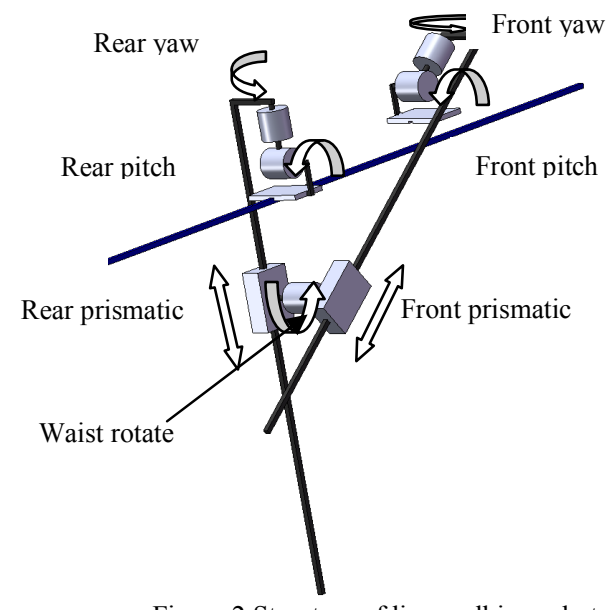

Figure 2 Structure of line-walking robot

To overcome these obstacles on power transmission line a novel biped line-walking mechanism was designed in [11]. As shown in figure 2, the line-walking mechanism features a biped structure and supported by two feet which can keep their grip on wires. Both of the feet can be set down or lifted off wires by the joint motion of the robot, and together with the alternating foot movement the robot can realize line-walking locomotion. The biped robot consists of $7 \mathrm{DOF}$, 2 DOF in each ankle, 1 DOF in each leg and 1 DOF in the waist. The robot has two prismatic joints to adjust the length of the length of the two legs respectively, and the centroid of the robot can be adjusted to the leg hanging from the wires during the single-support phase, thus the robot can reliably realize stable walking locomotion.

\section{B. Mechanical Structure of Power Transmission line Inspection Robot}

As considering power consumption and speed of displacement, the best approach to locomotion for power transmission line inspection is rolling on the conduct. Therefore a wheel driven system is developed to replace the foot at the end of each leg to build a practical power transmission line inspection robot.Figure 3 illustrated the structure of practical power line inspection robot.

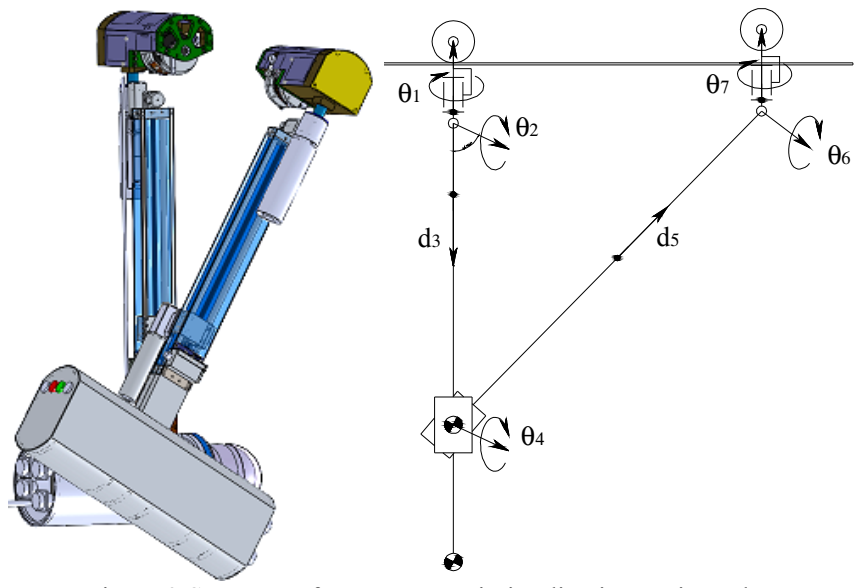

Figure 3 Structure of power transmission line inspection robot

To make sure that the robot will keep stable during power off time, all the joints are designed with the self-locking characteristic. Prismatic joint are realized with Acme screw assemble, and rotation joins are dived with worm gears. The payload of inspection are divided into two parts according to their function, left part called operation box and the right part is control box. With the precise design the weight of the two boxes are equal, which means the centre of weight of the two legs are at the same position.

\section{LOCOMOTION ANALYSIS}

\section{A. Rolling on Non-obstacle section}

Since the power line is hung between two poles, it forms a curve called a catenary. The line inclination angle with respect to the horizontal plane is changed when the robot rolling on the line. Thus the contact forces or even the contact state of the two wheels and the line will be changed according to the changing inclination angle. 


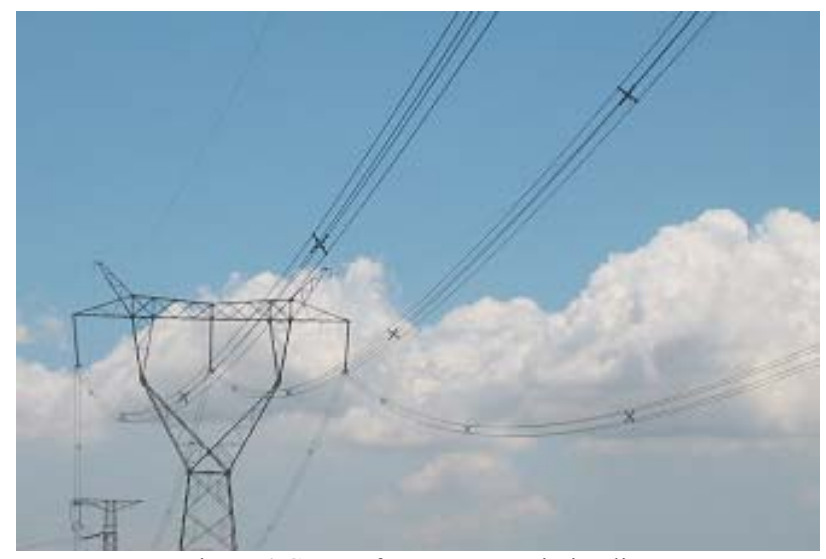

Figure 4 Curve of power transmission line

Generally the robot will be formed as a bilateral symmetry structure, as illustrated in figure 5.1, and the gravity central of the robot will located on the line which is perpendicular to line segment between the two wheels. Thus the normal pressure on the two wheels are equal when the robot running on the horizontal section, which means the max traction forces of the two wheels are same and the robot is in a relatively stable state.

While with the changing line inclination angle, as shown in figure 5, the contact forces or even the contact state of the two wheels and the line will be changed. In figure 5.2, the contact force of left wheel is less than that of the right wheel since the gravity central has moved to the right side, and eventually the left wheel lifted when the inclination angle reach the limit value, as shown in figure 5.3.

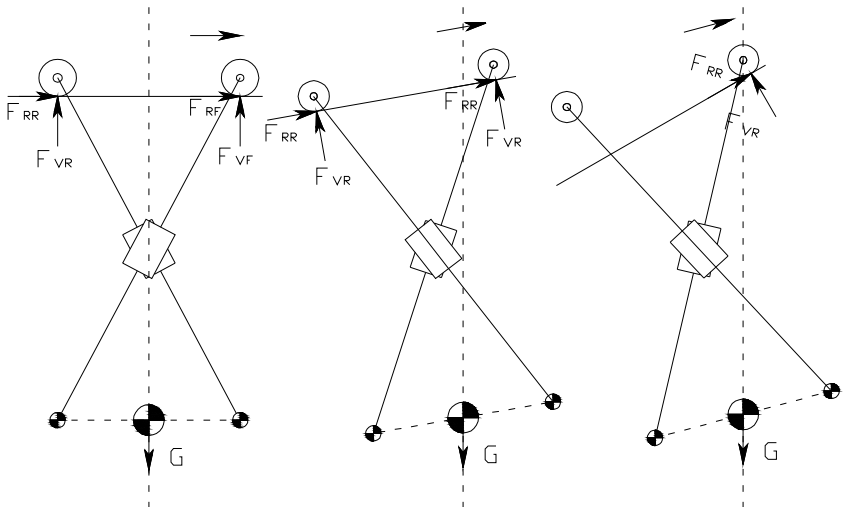

(1) horizontal line (2)less inclination line (3) large inclination line Figure 5 Forces and states analysis with different inclination angle

So the pose of the robot need to be adjusted to eliminate the load difference of the two wheels due to the changing inclination angle. Considering the highly nonlinear behavior of the robot and the complex of establishing precise statics analysis equations, a simplified model was used to decrease the computational time of the controller.

Figure 6 illustrates pose adjustment of the robot during the process of rolling on an inclined line. Assume the hip joint located at the central of the line segment between wheels and the weight central of both legs. The two prismatic joints were selected as the active joint to adjust the pose of the robot. When the two prismatic joint move at same speed but in position direction, the quadrilateral $W_{R F} W_{L F} G_{R F} G_{L R}$ formed as an isosceles trapezoid and the line segment between gravity central point and the middle point of the two wheels is parallel to two opposite sides of the isosceles trapezoid. Thus when the intersection angle of the non parallel side is twice the inclination angle of the power line the weight central of the robot is just below the middle point of the two wheels. Followings are detailed calculation process.

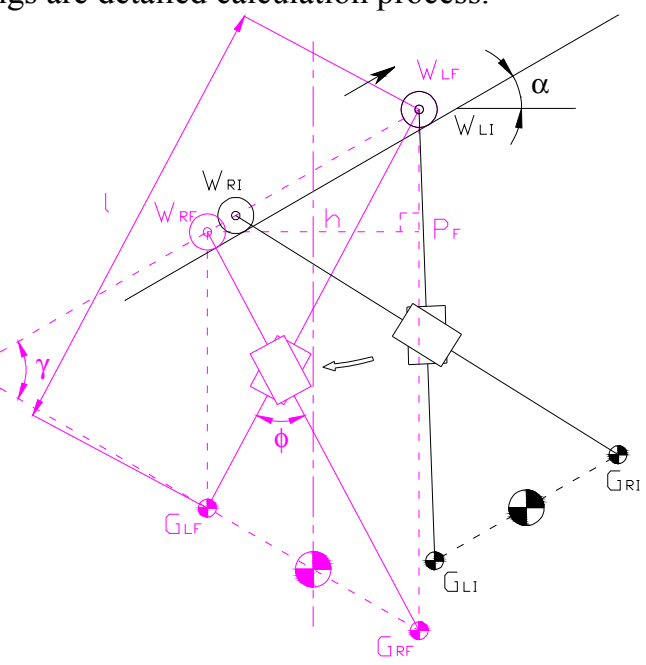

Figure 6 Pose adjustment process

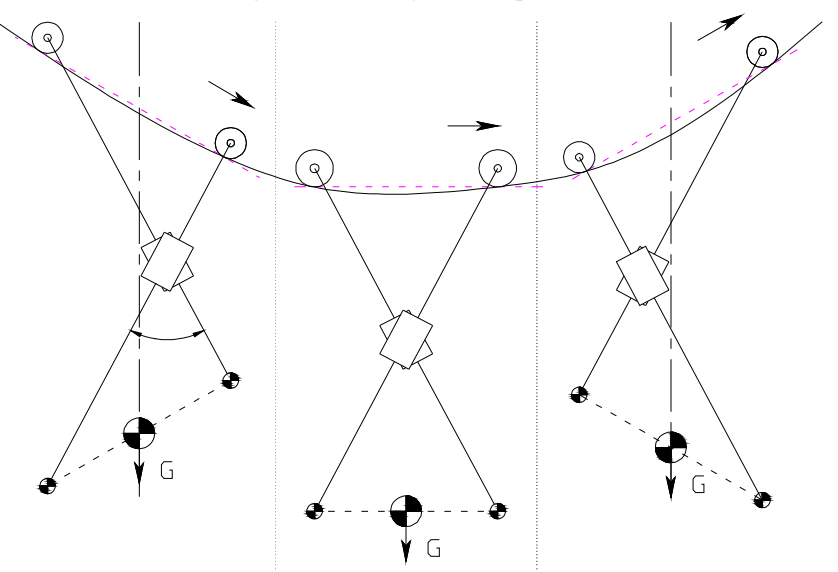

Figure 7 Power transmission line inspection robot based on novel line walking mechanism

With detected inclination angle, the variables of the two prismatic joints can be calculated using geometry.

$$
\angle W_{L F} W_{R F} P_{F}=\arctan \frac{W_{L F} P_{F}}{W_{R F} P_{F}} .
$$

In (1) $W_{R F} P_{F}$ represents height of the isosceles trapezoid, which equals to the length of a line segment between $W_{R F} G_{L F}$ and $W_{L F} G_{R F}$ that is perpendicular to them, $P_{F}$ is the perpendicular foot. With standard algebraic and trigonometric techniques, $W_{R F} P_{F}$ and $W_{L F} P_{F}$ can be found using

$$
\begin{gathered}
W_{R F} P_{F}=l \sin \frac{\varphi}{2} . \\
W_{L F} P_{F}=2 \Delta l \cos \frac{\varphi}{2} .
\end{gathered}
$$

Where $\Delta l$ is the displacement of the prismatic joint. 
While the gravity line is vertical we have

$$
\angle W_{L F} W_{R F} P_{F}=\alpha \text {. }
$$

By plunging (2),(3),(4) into (1) we have

$$
\Delta l=\frac{l}{2} \tan \frac{\varphi}{2} \tan \alpha .
$$

Thus with the known inclination angles the gravity central can be adjusted to the ideal position. Figure 7 is the sketch when the robot moves on a catenary with changing pose.

\section{B. Obstacle-Navigation}

Based on the morphology of the biped power line inspection robot, at least one wheel must remain in contact with the line all time, thus an obstacle-navigation cycle can be realized by combining single-supported phase and double-supported phase alternatively. Obstacle-navigation process is achieved by anchoring one foot to the line and swinging the other foot.

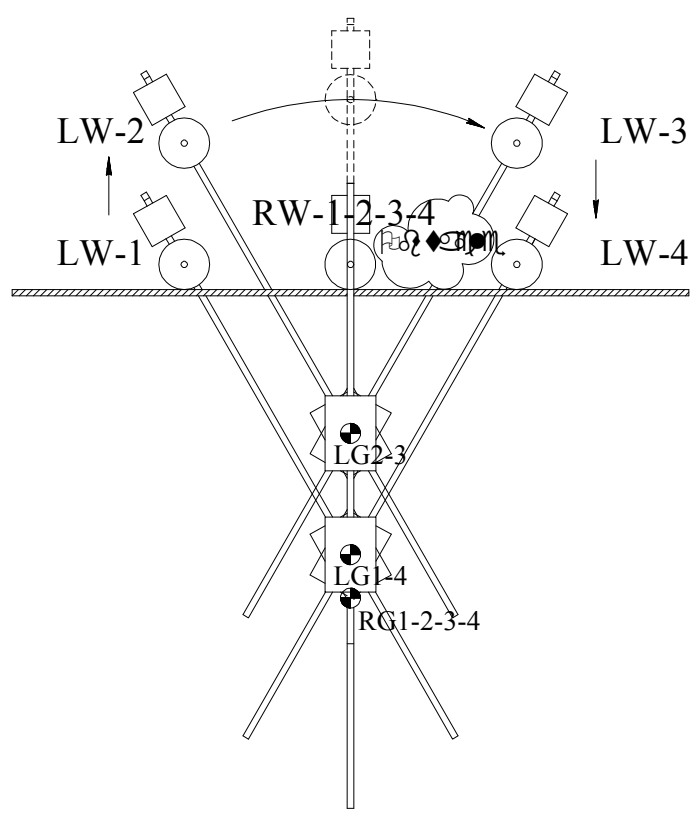

Figure 8 Obstacle-navigation process (first single support phase)

According to the structure of the power transmission line system obstacles can be divided into two types: lower obstacles like counter-weight and higher obstacles like clamps. For lower obstacles, obstacle-navigation can be realized by the flipping of legs.

Assuming that the robot is initially in the double-supported phase and both of the central gravities of the two legs locate at the right leg. During the single-supported phase, the right leg keeps sticking on the line vertically and the swing foot is lifted and flipped from the rear to the front of the fixing foot. The following sequence of motion can be executed that would result in the robot moving forward.

1. Contract right prismatic joint to make sure that the left wheel could cross the obstacle.
2. Rotate hip joint angle till the left wheel overcome the obstacle, and rotate left pitch joint to angle which will provide correct left wheel orientation.

3. Expand right prismatic joint to place the left wheel at LW-4

With the completion of this section, the left wheel of the robot has moved forward distance in straight line and overcome the obstacle. A double-supported phase then follows the single-supported phase to adjust the central of gravity from the right leg to the left leg which is essential to start the following single-supported phase. Adjusting is achieve by performing the following sequence and is illustrated in figure 9.

4. Expand right prismatic joint and contract left prismatic joint to adjust the central of gravities of the two legs from the right leg to the left leg and make sure the left leg is vertical, at the same time, three joints: R-yaw joint ,L-yaw joint and hip joint should rotate at the same time to keep the orientations and position of the two foot fixed.

5. The robot is now again in the beginning of single-supported phase.

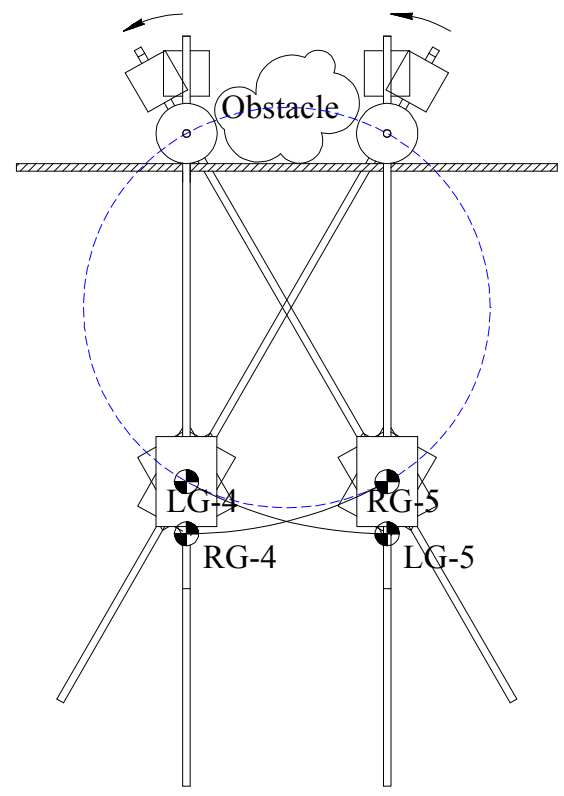

Figure 9 Obstacle-navigation process (double support phase)

It should be mentioned that in order to reduce the complexities of the controller only the two prismatic joints are actively controlled in the double support phase and the angle of hip joint is locked at a constant, which means, according to the geometry technology, the hip joint is moved on the circum circle of $R_{W} L_{W} H$ as shown in figure 9.

Thus after step 5 the robot return to the initial state with left wheel finishing obstacle-navigation, the following process is no different between steps 1-2-3 as shown in figure 9, except that the two wheels alternated. The following sequence of motion can be executed that would result in the completing of the obstacle-navigation. 
6. Contract left prismatic joint to make sure that the right wheel could cross the obstacle.

7. Rotate hip joint angle till the right wheel overcome the obstacle, and rotate right pitch joint to angle which will provide correct left wheel orientation.

8. Expand left prismatic joint to place the right wheel at RW-8

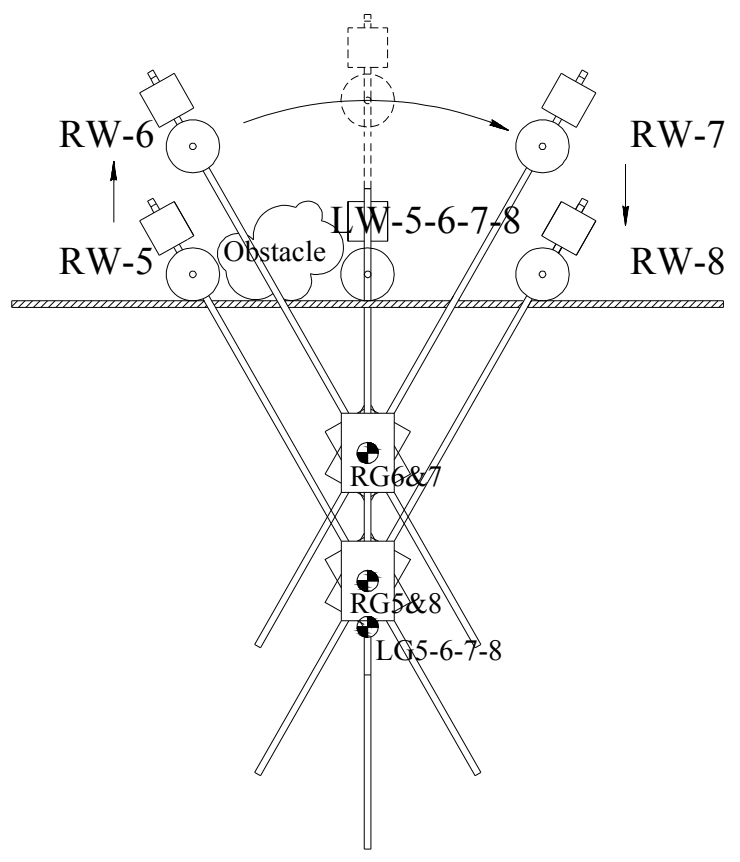

Figure 10 Obstacle-navigation process (final single support phase)

With respect to the higher obstacles, the swinging foot cannot stride over it, which means certain steps need to be added to the single-support phase. Thus before step 2 in the single support phase, the right pitch joint should be rotate to avoid the obstacle and after step 2 the right pitch joint should be rotated to the inverse direction to set the wheel on the line. Figure 11 illustrated the added step.

\section{CONTROL SYSTEM AND EXPERIMENTS}

As during the power line inspection operation there is a long distance between the operator and the inspection robot, wireless tele-operate structure was adopted to build the control system, as shown in figure 11 , which can be divided into two major subsystems: the on-board electronics installed in side the robot, and the ground control unit used by the operator.

\section{A. Control system of inspection robot}

The on-board electronic subsystem is described using figure 11. The robot is controlled by SCM/SPT2F, a miniaturized modular PC/AT compatible CPU board with 6 serial ports to communicate with the host computer and send motion commands to the servo driver module. Its operating system is RT-Linux, which enables the execution of a real-time processor. This feature of RT-Linux is essential for identical controllers of robot systems. The control software of the wire-walking robot has two modules. One module is used to communicate with the host computer and send motion commands to the servo driver, the other module is used to carry out the computations based on the kinematics equation to control the joints' trajectories to realize wire-walking locomotion. Expansion cards were selected to fulfill function to link the sensors signals to the central electronics.

All the eleven motors and their encoders are linked to the on-board electronic by two axe driver modules. The driver module is based on the TMS320F2812 digital signal processor chip from TI, which is suitable due to its support multi-motor control, low power consumption and its high-speed performance. Each DSP controller has two built-in quadrature encoder pulse (QEP) circuits to read the encoder of the servomotor; thus with each chip, two motors can be controlled with the encoder feedback. The total servo motor number of the wire-walking robot is 7 , which means at least 4 driver modules are needed. The control program is written in $\mathrm{C}$ language and the servo rate is $1 \mathrm{~Hz}$.

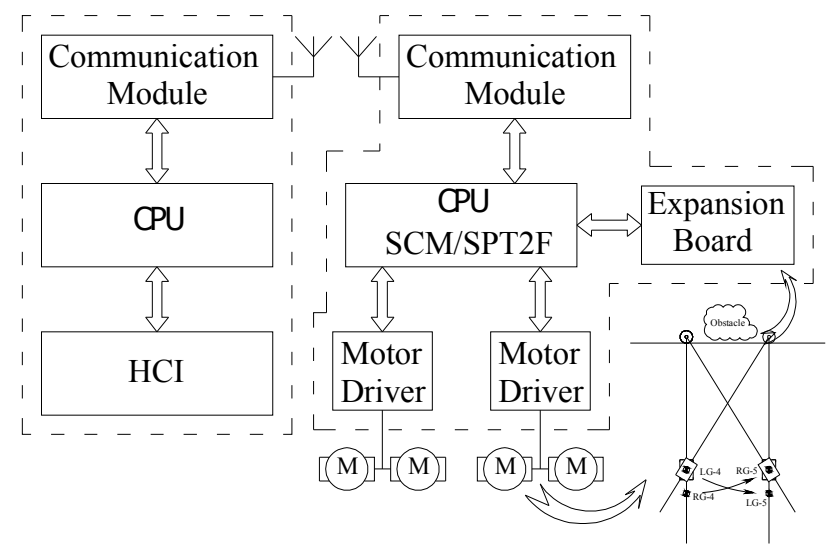

Figure 11 Architecture of the control system

The ground control unit consists of a embedded computer and two joysticks to control the moving speed of the robot and the pose of inspection camera. Control software was developed on $\mathrm{VC}++$ software. A display unit can show the video transferred form the robot for the operator to monitor state of robot and receive some inspection images.

\section{B. Experiment}

Experiments were conducted to verify the power transmission line inspection robot which was developed in this study. An indoor power transmission line environment was built to simulate the real condition. There are two counterweights, single overhang anchor clamp in the environment.

Figure 13 denotes that robot was hang on the power transmission line, with the algorithm provided in II the robot can adjust its position properly to ensure that the robot maintain in a relative stable state. 


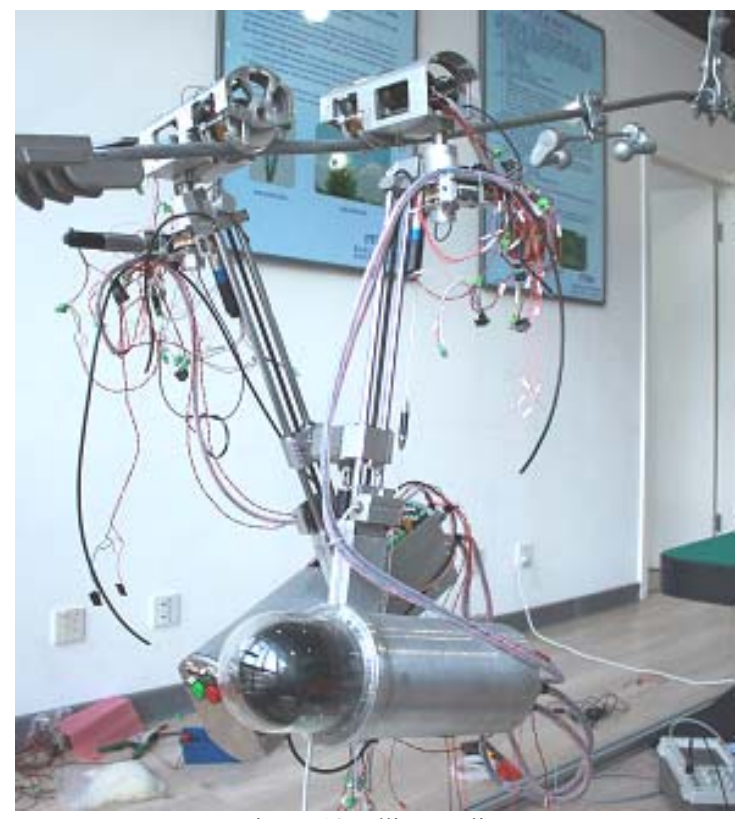

Figure 13 rolling on line

As illustrated in figure 14, when the robot encountered the obstacle-clamp it stopped at the line. The gravity central adjusted to the leg hanging on line, thus the other leg can be uplifted and swing from back to forward to overcome the obstacle.

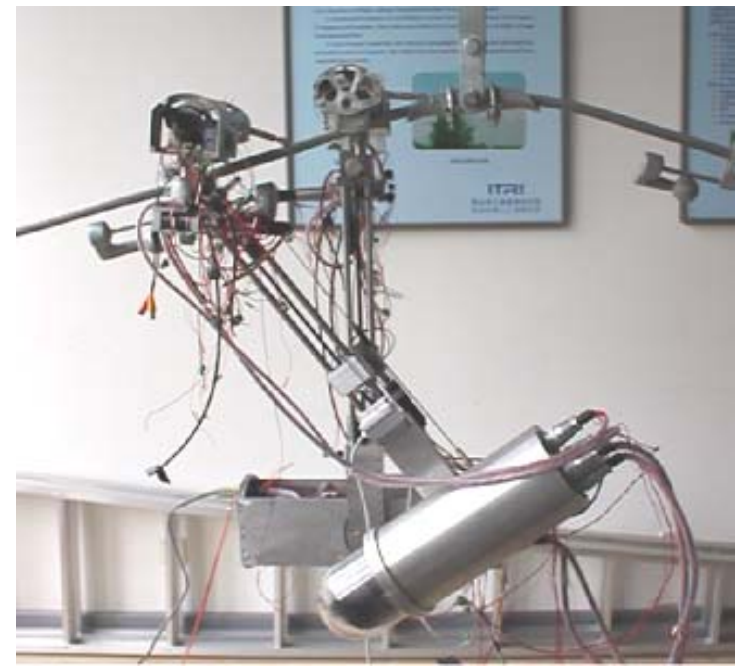

Figure 14 Obstacle-navigation process

\section{CONCLUSION}

A practical power transmission line inspection robot based on a novel line-walking mechanism is proposed in this paper. Combined with wheels and line-walking mechanism, the inspection robot can realize rolling on non-obstacle section efficiently and surmounting obstacles with articulated legs. In order to maintain stable state when rolling on line with changing inclination angles, pose adjustment algorithm was discussed in detail. The process of obstacle-navigation can be achieved by the combination robot locomotion which can be divided into two states: single-support phases and double support phase. To overcome different types of obstacles, certain steps have been added in the single-support phase.
After a brief introduction of the control system, experiments results are carry out to show the methods provided above are available. The future work will be focused on field experiments.

\section{REFERENCES}

[1] Jun Sawada,Kazuyuki Kusumoto,Tadashi Munakata,et al. A mobile robot for inspection of power transmission lines. IEEE Transactions on Power Delivery, vol.6, no. 1, pp.:309-315,1991.

[2] Toussaint,K Montambault, S., Pouliot, N.Transmission Line Maintenance Robots Capable of Crossing Obstacles: State-of-the-Art Review and Challenges Ahead. Journal of Field Robotics 26(5), 477-499 (2009)

[3] Montambault, S., Pouliot, N., Design and Validation of a Mobile Robot for Power Line Inspection and Maintenance. Field and Service Robotics, STAR 42, pp. 495-504, 2008.

[4] Montambault, S., \& Pouliot, N. (2008, June). The LineScout technology: Considerations for multi-span robotic teleoperated live-line inspection. In Proceedings of 9th International Conference on Live Maintenance (ICOLIM 2008), Torun, Poland.

[5] Nayyerloo, M., Yeganehparast, S., Barati, A., \& Foumani, M. (2007). Mechanical implementation and simulation of monolab, a mobile robot for inspection of power transmission lines. International Journal of Advanced Robotic Systems, 4(3), 381-386.

[6] Liang, Z., Li, E., \& Tan, M. (2005, August). Design and control for a tribrachiation mobile robot for the inspection of power transmission lines. In Proceedings of the Society of Instrument and Control Engineers Annual Conference (SICE 2005), Okayama, Japan (pp. 505-509).

[7] Tang, L., Fang, L., \& Wang, H. (2004b, December). Obstacle-navigation control for a mobile robot suspended on overhead ground wires. In Proceedings of International Conference on Control, Automation, Robotics and Vision (ICARCV 2004), Kunming, China (pp. 2080-2087).

[8] Wang, L., Fang., L., Wang, J. H., \& Zhao, M. (2006, October). Development and control of an autonomously obstacle-navigation inspection robot for extra-high voltage power transmission lines. In Proceedings of International Joint Conference (SICE-ICASE 2006), Bexco, Busan, Korea (pp. 5400-5405).

[9] Cai, L., Liang, Z., Hou, Z. G., \& Tan, M. (2008, April). Fuzzy control of the inspection robot for obstaclenegotiation. In Proceedings of IEEE International Conference on Networking, Sensing and Control (ICNSC2008), Sanya, China (pp. 117-122).

[10] Debenest, P., Guarnieri, M., Takita, K., Fukushima, E., Hirose, S., Tamara, K., Kimura, A., Kubokawa, H., Iwama, N., \& Shiga, F. (2008, May). Expliner-Robot for inspection of transmission lines. In Proceedings of the IEEE International Conference on Robotics and Automation (ICRA 2008), Pasadena, CA (pp. 3978-3984).

[11] Ludan Wang, Sheng Cheng, Jianwei Zhang: Development of a line-walking mechanism for power transmission line inspection purpose. IROS 2009: 3323-3328 